\title{
The initial assessment of expert panel performance in core hospitals for cancer genomic medicine in Japan
}

\author{
Kuniko Sunami ${ }^{1}$ - Yoichi Naito ${ }^{2}$ Eriko Aimono ${ }^{3} \cdot$ Toraji Amano $^{4} \cdot$ Daisuke Ennishi $^{5} \cdot$ Hidenori Kage $^{6} \cdot$ Masashi Kanai $^{7}$. \\ $K_{\text {Keigo Komine }}^{8} \cdot$ Takafumi Koyama $^{9} \cdot$ Takahiro Maeda $^{10}$. Sachi Morita ${ }^{11}$. Daisuke Sakai ${ }^{12}$. Shinji Kohsaka ${ }^{13}$. \\ Katsuya Tsuchihara ${ }^{14} \cdot$ Takayuki Yoshino $^{15}$
}

Received: 28 October 2020 / Accepted: 9 November 2020 / Published online: 1 January 2021

(c) The Author(s) 2021

\begin{abstract}
Background Since June 2019, cancer genomic profiling (CGP) tests have been reimbursed by the National Health Insurance system in Japan, with restrictions for government-designated hospitals with a molecular tumor board composed of multidisciplinary specialists, known as an expert panel (EP). The standardization of EPs is a critical challenge for implementing precision oncology in the clinical setting.

Methods Data on consecutive cases who underwent the CGP tests at 11 core hospitals between June 2019 and January 2020 were collected. We evaluated the proportions of cases that received genomically matched treatments, including investigational new drugs (INDs) based on CGP results, and/or for which genetic counseling was recommended. Two simulated cases were annotated by each EP. The annotated reports were then centrally assessed.

Results Each EP mainly discussed the applicability to genomically matched treatments and the necessity of performing genetic counseling. A pre-review of the report by key members in each EP reportedly made the EP conference more interactive and efficient, and thereby saved time. A total of 747 cases underwent CGP tests, 28 cases (3.7\%) received genomically matched treatment, and 17 cases (2.3\%) were referred for genetic counseling. Annotated reports for the simulated cases varied across the EPs, particularly the number of recommended IND trials, which seemed to be associated with the actual number of participants in IND trials.

Conclusions This investigation provides reference data for the application of precision oncology in a clinical setting. Further investigations on the standardization of clinical annotations are warranted.
\end{abstract}

Keywords Precision oncology · Comprehensive genomic profiling tests · Expert panel · Genetic counseling · Investigational new drug trials $\cdot$ Core hospitals

\section{Introduction}

Marked advances in precision oncology over the past two decades have made genotyping mandatory for most advanced cancer patients, as it helps to ensure proper

Kuniko Sunami and Yoichi Naito have contributed equally to this work.

Supplementary Information The online version contains supplementary material available at https://doi.org/10.1007/s1014 7-020-01844-1.

Takayuki Yoshino

tyoshino@east.ncc.go.jp

Extended author information available on the last page of the article therapy selection. Indeed, the National Comprehensive Cancer Network (NCCN) and European and Japanese Societies of Medical Oncology (ESMO and JSMO) practice guidelines now include recommendations for genotyping to guide therapy selection in at least 16 different types of cancer [1-3]. Despite this impressive progress, the pace of precision oncology innovations remains limited due to the daunting logistical realities of patient identification: many actionable targets are present in only a small fraction of patients, which means that hundreds or even thousands of patients need to be screened.

With the aim of implementing precision oncology in Japan, reimbursement has been provided by the National Health Insurance System for two comprehensive genomic profiling (CGP) tests, the OncoGuide NCC Oncopanel System [4] and 
the FoundationOne CDx Cancer Genomic Profile [5], since June 2019 [6]. The indication is restricted to patients with advanced solid tumors with disease progression during standard therapies or for whom there are no appropriate standard treatments, including rare cancers and carcinoma of unknown primary [6, 7]. In addition, for the purpose of consolidating precision oncology with quality control/assurance in designated hospitals, the Ministry of Health, Labour and Welfare (MHLW) originally designated 11 cancer genomic medicine core hospitals and 100 cooperative hospitals in April 2018 [8]. Reimbursement for CGP tests is restricted to these designated hospitals $[6,9]$.

Furthermore, before CGP test results are provided to the patient by an attending physician, these must be annotated by a regularly held intra-institutional molecular tumor board, called an expert panel (EP), which consist of multidisciplinary specialists at each core hospital, including medical oncologists, pathologists, genome researchers, medical geneticists, and genetic counselors, which are mandatory for a hospital to be designated as a core hospital. On the other hand, an attending physician working in a cooperative hospital should participate in regular EP meetings at the core hospital to which it is tied [6]. The EP meeting evaluates the pathogenicity of detected variants, the recommendations of genomically matched treatments, including investigational new drugs (INDs) and/or the necessity of genetic counseling based on the results of CGP tests $[6,10]$. Because clinical annotations made by the EPs may potentially affect the patient's actual treatment, the standardization of the EPs with quality control/assurance would be needed for implementing precision oncology in Japan.

In addition, the National Cancer Center (NCC) established the Center for Cancer Genomics and Advanced Therapeutics (C-CAT) in June 2018, which aggregates both the genomic data and clinical information of all patients who receive CGP tests in the clinical practice setting, to establish a central repository system [11]. The C-CAT also provides all of the core and cooperative hospitals with a clinical annotation report, called "C-CAT Findings" for each patient, which potentially make it easier to identify genomically matched treatments and to support the standardization of clinical annotation across EPs. However, the differences in clinical annotation among EPs have not been evaluated. We, therefore, conducted a survey of EPs at each core hospital and assessed the performance of EPs using two simulated cases.

\section{Methods}

\section{Composition of membership and survey to investigate the operation and performance of EPs}

The investigators, who were representatives of each EP at all of the core hospitals, are nominated. On February 27, 2020, an investigator meeting was held and the operation and performance of EPs was discussed. As a pre-meeting survey, we collected data on consecutive cases in which CGP tests were performed at all core hospitals between June 2019 and January 2020 and evaluated the number of cases that received genomically matched treatments and in which patients were referred for genetic counseling according to the recommendation by the EP. After consultation with the NCC Institutional Review Board (IRB), the IRB officially confirmed that the current study does not require IRB approval.

\section{Simulated case preparation}

Two simulated cases were developed by medical oncologists (KS, YN and TY) at the National Cancer Center Hospital (NCCH) and National Cancer Center Hospital East (NCCHE), which are the most experienced core hospitals and which have performed the largest number of CGP tests thus far.

Case 1 was a male with advanced colorectal cancer who had failed to respond to standard chemotherapies. The patient harbored the following somatic genetic alterations as follows: $B R A F \mathrm{~V} 600 \mathrm{E}, A T M$ R35*, NF1 Y1521*, $T P 53 \mathrm{R} 273 \mathrm{H}, A P C$ c. $1312+1 \mathrm{G}>\mathrm{A}, A R A F \mathrm{R} 326^{*}$ and NTRK2 L138P, and BRCA2 V208G germline variant. Case 2 was a female with advanced breast cancer whose disease progressed on standard therapies, who showed PIK3CA H1047R, ERBB2 S310Y and CCND1 amplification. Cases 1 and 2 were tested by the OncoGuide NCC Oncopanel System and FoundationOne CDx Cancer Genomic Profile, respectively (Table 1). Simulated CGP result reports of these two cases were prepared by the medical oncologists (KS, YN, and TY), and simulated C-CAT Findings were prepared by C-CAT. Then, these reports (clinical history, results of the simulated CGP test and simulated C-CAT findings) were delivered to all EPs at core hospitals on December 24, 2019.

\section{Annotation by EPs for simulated cases}

Each EP discussed the simulated CGP test results and simulated C-CAT findings and made recommended genomically matched treatment according to the evidence level and necessity of genetic counseling during their routine EP meetings, between December 25, 2019 and January 31, 2020. Note that the developers of simulated cases (KS, YN, and TY) never joined in the discussion at the EP meetings for these cases. The evidence level was defined in "Consensus clinical practice guidance for the CGP tests," which was issued by three cancer-related major Japanese societies in October 2017 [12]. The annotated reports from each EP were centrally assessed by the developers (KS, YN, and TY) before the investigator meeting held on February 27, 2020. 
Table 1 Clinical information and the CGP results of simulated cases

\begin{tabular}{|c|c|c|}
\hline & Case 1 & Case 2 \\
\hline Gender & Male & Female \\
\hline Age & $50 \mathrm{~s}$ & $40 \mathrm{~s}$ \\
\hline Tumor type & Colon cancer & Breast cancer $(\mathrm{ER}+, \mathrm{PgR}+, \mathrm{HER} 21+)$ \\
\hline \multicolumn{3}{|l|}{ Previous chemotherapies } \\
\hline 1st line & FOLFOX + bevacizumab & Anastrozol \\
\hline 2nd line & FOLFIRI + cetuximab & Fulvestrant + palbociclib \\
\hline 3rd line & Investigational drug & Paclitaxel + bevacizumab \\
\hline 4th line & Regorafeinib & Eriblin \\
\hline 5th line & - & Capecitabin \\
\hline 6th line & - & Doxorubicin + cyclophosphamide \\
\hline \multirow[t]{2}{*}{ Family history of cancer } & Mother: breast cancer (40s) & N/A \\
\hline & Sister: breast cancer (50s) & \\
\hline Medical history & Hypertension & N/A \\
\hline Type of GCP test & OncoGuide ${ }^{\mathrm{TM}} \mathrm{NCC}$ oncopanel & FoundationOne ${ }^{\circledR} \mathrm{CDx}$ \\
\hline \multirow[t]{10}{*}{ Detected SNVs and CNVs } & Somatic variants & \\
\hline & $B R A F \mathrm{~V} 600 \mathrm{E}$ & $P I K 3 C A$ H1047R \\
\hline & ATM R35* & $E R B B 2 \mathrm{~S} 310 \mathrm{Y}$ \\
\hline & $N F 1 \mathrm{Y} 1521 *$ & CCND1 amplification \\
\hline & TP53 R273H & \\
\hline & $A P C$ c. $1312+1 \mathrm{G}>\mathrm{A}$ & \\
\hline & ARAF R326* & \\
\hline & NTRK2 L138P & \\
\hline & Germline variant & \\
\hline & BRCA2 V208G & \\
\hline
\end{tabular}

N/A not applicable, $C G P$ comprehensive genomic profiling, $S N V$ single-nucleotide variant, $C N V$ copy number variant, FOLFOX oxaliplatin, folinic acid and 5-fluorouracil, FOLFIRI irinotecan, folinic acid and 5-fluorouracil, BRAF V-Raf murine sarcoma viral oncogene homolog $\mathrm{B} 1, A T M$ ataxia telangiectasia mutated, NF1 neurofibromatosis type I, TP53 tumor protein p53, APC adenomatous polyposis coli, ARAF V-Raf murine sarcoma 3611 viral oncogene homolog 1, NTRK2 neurotrophic tyrosine receptor kinase 2, $B R C A 2$ breast cancer susceptibility gene 2, PIK3CA phosphoinositide-3-kinase, catalytic, alpha polypeptide, ERBB2 human epidermal growth factor receptor 2, CCND1 cyclin D1

\section{Results}

\section{The operation of EPs at each core hospital}

At the investigator meeting, investigators from each EP demonstrated their daily work at the EP and we recognized that all of the EPs mainly discussed the applicability to genomically matched treatment, including IND trials, and the necessity of genetic counseling. In addition, for all investigators, it was suggested that all pre-reviewing would be carefully carried out by the key members of each EP to make the conference more interactive and to also save time.

\section{The performance of EPs at each core hospital}

A total of 1522 cases (core hospitals, $n=747$; cooperative hospitals, $n=775$ ) underwent CGP tests from June 2019 to January 2020. Among the 747 cases in core hospitals, 28 cases $(3.7 \%$, range $0-7.6 \%)$ received genomically matched treatments (Table 2); 16 of the 28 cases (2.1\%) participated in the IND trials and three cases $(0.4 \%)$ received genomically-matched treatment as an off-label treatment. The remaining eight cases $(1.0 \%)$ received approved moleculartargeted drugs. The most common treatments were HER2 inhibitors for $E R B B 2$ alterations $(n=6)$, followed by FGFR inhibitors for $F G F R / F G F$ alterations $(n=4)$, EGFR inhibitors for $E G F R$ mutations ( $n=2)$, NTRK inhibitors for NTRK fusions and mTOR inhibitors for PIK3CA mutations $(n=2)$ (Supplementary Table 1). Regarding the germline findings, 17 of 747 cases (2.3\%, range $0-15 \%)$ were referred for genetic counselling (Supplementary Table 2).

\section{Clinical annotations of Case 1 (Table 3)}

Nine of 11 EPs recommended dabrafenib (BRAF inhibitor) and trametinib (MEK inhibitor) combination therapy for a $B R A F$ V600E mutation. Triplet therapy with encorafenib (BRAF inhibitor), binimetinib (MEK inhibitor), and 
Table 2 The performance of each expert panel

\begin{tabular}{lrll}
\hline Core hospital & $\begin{array}{l}\text { No. of patients underwent } \\
\text { the CGP test }\end{array}$ & $\begin{array}{l}\text { No. of patients received } \\
\text { "matched" therapies }\end{array}$ & $\begin{array}{l}\text { No. of patients } \\
\text { referred to genetic } \\
\text { counseling }\end{array}$ \\
\hline A & 75 & 3 & 3 \\
B & 60 & 2 & 0 \\
C & 5 & 0 & 0 \\
D & 41 & 0 & 1 \\
E & 160 & 16 & 5 \\
F & 172 & 4 & 2 \\
G & 13 & 1 & 2 \\
H & 13 & 0 & 0 \\
I & 98 & 0 & 0 \\
J & 24 & 0 & 2 \\
K & 86 & 2 & 3 \\
Total & 747 & $28(3.7 \%)$ & $18(2.4 \%)$ \\
\hline
\end{tabular}

cetuximab was recommended by four sites (Sites C, E, I and $\mathrm{K}$ ) because the triplet/doublet (encorafenib plus cetuximab) regimens showed a survival benefit in pre-treated metastatic colorectal cancer patients with $B R A F$ V600E mutation in the BEACON global phase 3 trial $[13,14]$. Although the ANCHOR global phase 2 trial with the triplet regimen was ongoing in Japan at that time, the eligibility was restricted to chemotherapy-naïve patients; thus, the other seven sites did not recommend it [15]. TP0903, an AXL kinase inhibitor, was recommended by Sites F and I [16]. For ATM truncating mutation, two sites (Sites E and F) recommended BAY-1895344, ATR inhibitor [17], and Site E recommended talazoparib plus avelumab [18]. Regarding other candidate therapies, LXH254 (a pan-RAF inhibitor) [19], TP0903, TAK931 (a CDC7 inhibitor) [20], and olaparib (based on a phase 2 trial for advanced solid tumors) [21], and talazoparib + avelumab were considered by Site A, while trametinib was considered by Site F. Site G concluded that there was no recommendation and dabrafenib and trametinib therapy was considered as a treatment option. All 11 sites annotated BRCA2 V208G as a germline variant of uncertain significance (VUS). Considering the familial history of breast cancers, two EPs (Sites D and $\mathrm{H}$ ) recommended genetic counseling (Supplementary Table 3).

\section{Clinical annotations of Case 2 (Table 4)}

One site (Site D) recommended everolimus plus exemestane for PIK3CA mutation. That regimen was also considered as a treatment option by Sites A, F and J. Alpelisib or PI3K inhibitor, which are approved by the FDA for hormone receptor-positive breast cancer with $P I K 3 C A$ mutation [22],

Table 3 Clinical annotation for simulated case 1

\begin{tabular}{lll}
\hline Core hospital & Recommended therapy & Considered therapy \\
\hline A & Dabrafenib + trametinib & $\begin{array}{c}\text { LXH254, TP0903, olaparib, talazo- } \\
\text { parib + avelumab, BAY1895344, } \\
\text { TAK-931 }\end{array}$ \\
B & Dabrafenib + trametinib & - \\
C & Binimetinib + cetuximab + encorafenib & - \\
D & Dabrafenib + trametinib & - \\
E & Binimetinib + cetuximab + encorafenib, Dabrafenib + trametinib, talazoparib + ave- & - \\
F & lumab, BAY1895344 & Trametinib \\
G & Dabrafenib + trametinib, TP0903, BAY1895344 & Dabrafenib + trametinib \\
H & - & - \\
I & Dabrafenib + trametinib & - \\
J & Binimetinib + cetuximab + encorafenib, Dabrafenib + trametinib, TP0903 & - \\
K & Dabrafenib + trametinib & - \\
\hline
\end{tabular}

- not recommended/considered therapies 
were recommended by three sites (Sites $\mathrm{H}, \mathrm{I}$, and K), but not recommended by the other 8 sites due to closure of the clinical trial in Japan. Trastuzumab deruxtecan was recommended by two sites (Sites C and D) for HER2 IHC1+ [23]. No sites evaluated ERBB2 S310Y and CCND1 amplification as druggable. Two sites (Sites B and G) concluded that no genomically matched treatment was available for this patient (Supplementary Table 4).

\section{Discussion}

We reported the initial assessment of the performance of each EP at core hospitals. In the first 8 months after the implementation of reimbursement for CGP tests, 747 cases (ranging 5-172 cases) were tested at 11 core hospitals and $3.7 \%$ of these cases received genomically matched treatment. In terms of germline findings, $2.4 \%$ of tested cases were referred for genetic counseling. While more than half of these cases participated in IND trials, $11 \%$ of cases received matched drugs as an off-label treatment. The findings serve as reference data for assessing the improvement in precision oncology in Japan in the future. We also demonstrate that the clinical annotations of CGP tests varied across EPs, which seemed to be associated with the recognition of the latest information regarding the recruitment status and the eligibility of candidate for IND trials. Because such information cannot be tacked on by the C-CAT, it might be necessary to establish a framework to share the latest information of IND trials across all EPs. In the United States, a virtual molecular tumor board (VMTB), cloud-based computing technology for integrating the CGP results of each patient with a genomic knowledge base to provide an annotation report on candidate trials, showed clinical utility not only for standardizing clinical annotations but also for improving the efficacy

Table 4 Clinical annotation for simulated case 2

\begin{tabular}{lll}
\hline Core hospital & Recommended therapy & Considered therapy \\
\hline A & - & Everolimus + exemestane \\
B & - & - \\
C & Trastuzumab deruxtecan & - \\
D & Everolimus + exemestane, & - \\
& trastuzumab deruxtecan & \\
E & Trastuzumab deruxtecan & - \\
F & - & Everolimus + exemestane \\
G & - & - \\
H & Alpelisib & Afatinib \\
I & Alpelisib, niratinib & - \\
J & - & Everolimus + exemestane \\
K & PI3K inhibitor & - \\
\hline
\end{tabular}

- not recommended/considered therapies of on-site molecular tumor boards [24]. A VMTB may also be an option for Japan.

In previous reports of research-based genomic screening projects, $3-20 \%$ of patients received genomically matched treatment on the basis of their CGP test results [25-34]. Our study showed that $3.7 \%$ of patients received genomically matched treatment; this percentage seems to be lower in comparison to previous reports. One potential reason for this finding is due to the fact that the CGP tests were mainly reimbursed for patients who had no option other than to receive the best supportive care. Based on the data obtained from both NCI-MATCH and SCRUM-Japan GI SCREEN, nationwide genomic screening projects for refractory patients who have no systemic treatment options, the proportion of patients who enrolled in the matched clinical trials was $2.4 \%$ and $2.2 \%[34,35]$, which was similar to the proportion reported in our study. Fundamentally, in addition to refractory patients, precision oncology should also be applied to non-refractory patients. Recently, the "Consensus clinical practice guidance for CGP tests" was updated and now recommends CGP test would be recommended, regardless of the line of treatment $[36,37]$.

Our study has demonstrated the actual data related to the clinical utility of the CGP test in core hospitals in the clinical setting. However, one limitation associated with our study is due to the fact that the data from cooperative hospitals could not be evaluated since the information regarding the post-expert panel clinical course of cases in these hospitals was not available.

Along with the current increase in the number of CGP tests across Japan, in September 2019, the MHLW established a new designated hospital category, called hub hospitals, which are positioned between core and cooperative hospitals [38]. Hub hospitals are required to establish an EP. Thus, the total number of designated hospitals has recently been expanded to 12 core, 33 hub and 165 cooperative hospitals [38]. Considering the increased numbers of hospitals (core and hub) carrying out EPs, the standardization of clinical annotations has become more relevant to maintaining quality control/assurance of precision oncology in Japan.

We are conducting a further study to develop consensus clinical annotations using another 100 simulated cases covering genomic alterations that are observed with high frequency (approximately 70\% of clinical cases) with reference to The Cancer Genome Atlas (TCGA) database (accessed 2 April 2020) [39], as an official educational material for EPs across Japan, and hopefully worldwide. We will also assess the learning effect using this educational material. We believe that our further study will help with the standardization of all EPs.

In conclusion, this is an initial report on the performance of EPs in core hospitals after the implementation of reimbursement for CGP. This report is also the first to raise issues 
regarding the uniformity of the process and the quality of recommendations that have a great impact on decisionmaking by patients and attending physicians on EPs at core hospitals. Further investigation on the standardization of EPs is warranted.

Acknowledgements The authors are grateful to C-CAT and Mitsui Knowledge Industry Co., Ltd. for the creation of the mock C-CAT Findings of the simulated cases, which was undertaken on a voluntary basis. We also thank all EP members at 11 core hospitals, Hideki Ueno and Yuichiro Ohe for supporting this study. This study was supported by grants from the Ministry of Health, Labour and Welfare.

Funding This work was supported by The Ministry of Health, Labour and Welfare, Health Labour Sciences Special Research Grant (19EA1007).

\section{Compliance with ethical standards}

Conflict of interest No author has any conflict of interest.

Open Access This article is licensed under a Creative Commons Attribution 4.0 International License, which permits use, sharing, adaptation, distribution and reproduction in any medium or format, as long as you give appropriate credit to the original author(s) and the source, provide a link to the Creative Commons licence, and indicate if changes were made. The images or other third party material in this article are included in the article's Creative Commons licence, unless indicated otherwise in a credit line to the material. If material is not included in the article's Creative Commons licence and your intended use is not permitted by statutory regulation or exceeds the permitted use, you will need to obtain permission directly from the copyright holder. To view a copy of this licence, visit http://creativecommons.org/licenses/by/4.0/.

\section{References}

1. National Comprehensive Cancer Network Guidelines (2020). https://www.nccn.org/professionals/physician_gls/defau 1t.aspx. Accessed Aug 2020

2. El-Deiry WS, Goldberg RM, Lenz HJ et al (2019) The current state of molecular testing in the treatment of patients with solid tumors. CA Cancer J Clin 69:305-343

3. Yoshino T, Arnold D, Taniguchi $\mathrm{H}$ et al (2018) Pan-Asian adapted ESMO consensus guidelines for the management of patients with metastatic colorectal cancer: a JSMO-ESMO initiative endorsed by CSCO, KACO, MOS, SSO and TOS. Ann Oncol 29:44-70

4. The OncoGuide ${ }^{\mathrm{TM}} \mathrm{NCC}$ oncopanel system receives insurance coverage for use in cancer genome profiling. https://www.sysme x.co.jp/en/news/2019/190531.html. Accessed Aug 2020

5. Chugai Launches Genomic Mutation Analysis Program, FoundationOne CDx Cancer Genomic Profile. https://www.chugai-pharm .co.jp/english/news/detail/20190603150001_625.html. Accessed Aug 2020

6. Ebi H, Bando H (2019) Precision oncology and the universal health coverage system in Japan. JCO Precis Oncol. https://doi. org/10.1200/po.19.00291(3):1-12

7. https://www.mhlw.go.jp/content/12400000/000514782. pdf. Accessed Aug 2020

8. https://www.mhlw.go.jp/file/05-Shingikai-10901000-Kenkoukyok u-Soumuka/0000203236.pdf. Accessed Aug 2020
9. Sunami K (2019) The role of core hospitals and cooperative hospitals for cancer genomic medicine in Japan. Gan To Kagaku Ryoho 46:617-621

10. Malone ER, Oliva M, Sabatini PJB et al (2020) Molecular profiling for precision cancer therapies. Genome Med 12(1):8

11. National Cancer Center Center for Cancer Genomics and Advanced Therapeutics. https://www.ncc.go.jp/en/c_cat/about/ index.html. Accessed Aug 2020

12. Sunami K, Takahashi H, Tsuchihara K et al (2018) Clinical practice guidance for next-generation sequencing in cancer diagnosis and treatment (edition 1.0). Cancer Sci 109(9):2980-2985

13. Corcoran RB, Atreya CE, Falchook GS et al (2015) Combined BRAF and MEK inhibition with dabrafenib and trametinib in BRAF V600-mutant colorectal cancer. J Clin Oncol 33:4023-4031

14. Kopetz S, Grothey A, Yaeger R et al (2019) Encorafenib, binimetinib, and cetuximab in BRAF V600E-mutated colorectal cancer. N Engl J Med 381:1632-1643

15. Grothey A, Tabernero J, Taieb J et al (2020) ANCHOR CRC: a single-arm, phase 2 study of encorafenib, binimetinib plus cetuximab in previously untreated BRAF V600-mutant metastatic colorectal cancer. Ann Oncol Suppl 3:S242-243

16. First-in-human study of oral TP-0903 (a novel inhibitor of axl kinase) in patients with advanced solid tumors. https://clinicaltr ials.gov/ct2/show/NCT02729298. Accessed Aug 2020

17. First-in-human study of ATR inhibitor BAY1895344 in patients with advanced solid tumors and lymphomas. https://clinicaltrials. gov/ct2/show/NCT03188965. Accessed Aug 2020

18. Javelin BRCA/ATM: avelumab plus talazoparib in patients with BRCA or ATM mutant solid tumors. https://clinicaltrials.gov/ct2/ show/NCT03565991. Accessed Aug 2020

19. Phase I study of LXH254 in patients with advanced solid tumors haboring MAPK pathway alterations. https://clinicaltrials.gov/ct2/ show/NCT02607813. Accessed Aug 2020

20. A study to evaluate TAK-931 in participants with advanced nonhematologic tumors. https://clinicaltrials.gov/ct2/show/NCT02 699749. Accessed Aug 2020

21. Efficacy and safety of olaparib (MK-7339) in participants with previously treated, homologous recombination repair mutation (HRRm) or homologous recombination deficiency (HRD) positive advanced cancer (MK-7339-002 / LYNK-002). https://clinicaltr ials.gov/ct2/show/NCT03742895. Accessed Aug 2020

22. André F, Ciruelos E, Rubovszky G et al (2019) Alpelisib for PIK3CA-mutated, hormone receptor-positive advanced breast cancer. N Engl J Med 380:1929-1940

23. Trastuzumab deruxtecan (DS-8201a) versus investigator's choice for HER2-low breast cancer that has spread or cannot be surgically removed [DESTINY-Breast04]. https://clinicaltrials.gov/ct2/ show/NCT0373402921. Accessed Aug 2020

24. Pishvaian MJ, Blais EM, Bender RJ et al (2019) A virtual molecular tumor board to improve efficiency and scalability of delivering precision oncology to physicians and their patients. JAMIA Open 2:505-515

25. Naito Y, Takahashi H, Shitara K et al (2018) Feasibility study of cancer genome alterations identified by next generation sequencing: ABC study. Jpn J Clin Oncol 48:559-564

26. Sunami K, Ichikawa H, Kubo T et al (2019) Feasibility and utility of a panel testing for 114 cancer-associated genes in a clinical setting: a hospital-based study. Cancer Sci 110:1480-1490

27. Tanabe Y, Ichikawa H, Kohno T et al (2016) Comprehensive screening of target molecules by next-generation sequencing in patients with malignant solid tumors: guiding entry into phase I clinical trials. Mol Cancer 15:73

28. Kou T, Kanai M, Yamamoto Y et al (2017) Clinical sequencing using a next-generation sequencing-based multiplex gene assay in patients with advanced solid tumors. Cancer Sci 108:1440-1446 
29. Sohal DP, Rini BI, Khorana AA et al (2015) Prospective clinical study of precision oncology in solid tumors. J Natl Cancer Inst. https://doi.org/10.1093/jnci/djv332

30. Khoury JD, Wang WL, Prieto VG et al (2018) Validation of immunohistochemical assays for integral biomarkers in the NCIMATCH EAY131 clinical trial. Clin Cancer Res 24:521-531

31. NCI-MATCH precision medicine cancer trial. https://ecog-acrin .org/nci-match-eay131. Accessed Aug 2020

32. Meric-Bernstam F, Brusco L, Shaw K et al (2015) Feasibility of large-scale genomic testing to facilitate enrollment onto genomically matched clinical trials. J Clin Oncol 33:2753-2762

33. Trédan O, Wang Q, Pissaloux D et al (2019) Molecular screening program to select molecular-based recommended therapies for metastatic cancer patients: analysis from the ProfiLER trial. Ann Oncol 30:757-765

34. Flaherty KT, Gray R, Chen A et al (2020) The molecular analysis for therapy choice (NCI-MATCH) trial: lessons for genomic trial design. J Natl Cancer Inst. https://doi.org/10.1093/jnci/djz245

35. Nakamura Y, Taniguchi H, Bando $\mathrm{H}$ et al (2020) Clinical utility of circulating tumor DNA sequencing in advanced gastrointestinal cancer: SCRUM-Japan GI-SCREEN and GOZILA studies. Nat Med (in press)
36. Consensus clinical practice guidance for the CGP tests" issued by three cancer-related major Japanese societies (the Japanese Society of Medical Oncology, the Japanese Society of Clinical Oncology, and the Japanese Cancer Association) version 2.0. https ://www.jsmo.or.jp/about/kanko.html or http://www.jca.gr.jp/resea rcher/topics/2020/200310.html. Accessed Aug 2020

37. Yoshino T, Pentheroudakis G, Mishima S et al (2020) JSCOESMO-ASCO-JSMO-TOS: international expert consensus recommendations for tumour-agnostic treatments in patients with solid tumours with microsatellite instability or NTRK fusions. Ann Oncol 31:861-872

38. https://www.mhlw.go.jp/stf/seisakunitsuite/bunya/kenkou_iryou/ kenkou/gan/gan_byoin.html. Accessed Aug 2020

39. cBioPortal for cancer genomics. https://www.cbioportal .org/. Accessed Apr 2020

Publisher's Note Springer Nature remains neutral with regard to jurisdictional claims in published maps and institutional affiliations.

\section{Affiliations}

\section{Kuniko Sunami ${ }^{1}$. Yoichi Naito ${ }^{2}$ Eriko Aimono ${ }^{3} \cdot$ Toraji Amano $^{4} \cdot$ Daisuke Ennishi $^{5} \cdot$ Hidenori Kage $^{6} \cdot$ Masashi Kanai $^{7}$. $K_{\text {Keigo Komine }}^{8} \cdot$ Takafumi Koyama $^{9} \cdot$ Takahiro Maeda $^{10}$. Sachi Morita ${ }^{11}$. Daisuke Sakai ${ }^{12}$. Shinji Kohsaka ${ }^{13}$. Katsuya Tsuchihara ${ }^{14} \cdot$ Takayuki Yoshino $^{15}$}

1 Department of Laboratory Medicine, National Cancer Center Hospital, Tokyo, Japan

2 Department of Breast and Medical Oncology, National Cancer Center Hospital East, Kashiwa, Japan

3 Genomics Unit, Keio University School of Medicine, Tokyo, Japan

4 Clinical Research and Medical Innovation Center, Hokkaido University Hospital, Sapporo, Japan

5 Department of Hematology and Oncology, Okayama University Hospital, Okayama, Japan

6 Department of Respiratory Medicine, The University of Tokyo Hospital, Tokyo, Japan

7 Department of Therpeutic Oncology, Graduate School of Medicine, Kyoto University, Kyoto, Japan

8 Department of Clinical Oncology, Tohoku University Hospital, Sendai, Japan

9 Department of Experimental Therapeutics, National Cancer Center Hospital, Tokyo, Japan
10 Department of Hematology, Oncology and Cardiovascular Medicine, Kyushu University Hospital, Fukuoka, Japan

11 Department of Clinical Oncology and Chemotherapy, Nagoya University Hospital, Nagoya, Japan

12 Center for Cancer Genomics and Personalized Medicine, Osaka University, Suita, Japan

13 Section of Knowledge Integration, Center for Cancer Genomics and Advanced Therapeutics, National Cancer Center, Tokyo, Japan

14 Division of Translational Informatics, National Cancer Center Exploratory Oncology Research and Clinical Trial Center, Tokyo, Japan

15 Department of Gastrointestinal Oncology, National Cancer Center Hospital East, 6-5-1, Kashiwanoha, Kashiwa-shi, Chiba 277-8577, Japan 